\title{
First-pass and high-resolution ECG-gated MRA of the thoracic vasculature in children and adolescents using gadobutrol at $3 \mathrm{~T}$
}

Darius Dabir*, Claas Philip Naehle, Andreas Mueller, Katharina Strach, Hans Schild, Daniel Thomas

From 2011 SCMR/Euro CMR Joint Scientific Sessions

Nice, France. 3-6 February 2011

\section{Introduction}

Using first-pass MRA (FP-MRA) spatial resolution is limited by breath-hold duration, and image quality (IQ) may be hampered by motion artefacts. Studies at $1.5 \mathrm{~T}$ have shown that the use of blood-pool contrast agents (BP-CA) allows for motion-compensated high-resolution MRA during the steady-state, yielding significantly higher IQ compared to FP-MRA. However, MRA with $\mathrm{BP}-\mathrm{CAs}$ does not allow for viability imaging. At $3 \mathrm{~T}$ the use of a slowly injected extracellular CA at double dose may allow for a high-resolution MRA (HR-MRA) AND viability imaging.

\section{Purpose}

The purpose of this study was 1) to implement an ECGgated free breathhing HR-MRA protocol using an extracellular CA (Gadobutrol) at $3 \mathrm{~T}$ and 2) to compare the vessel sharpness and image quality of standard FP-MRA to HR-MRA of the thoracic vasculature in children and adolescents with congenital disorders (CD) or acquired disease $(\mathrm{AD})$ of the thoracic vasculature.

\section{Methods}

To date, 13 Patients (age range: 5 to 41 years) with CD or $\mathrm{AD}$ of the thoracic vasculature underwent both,

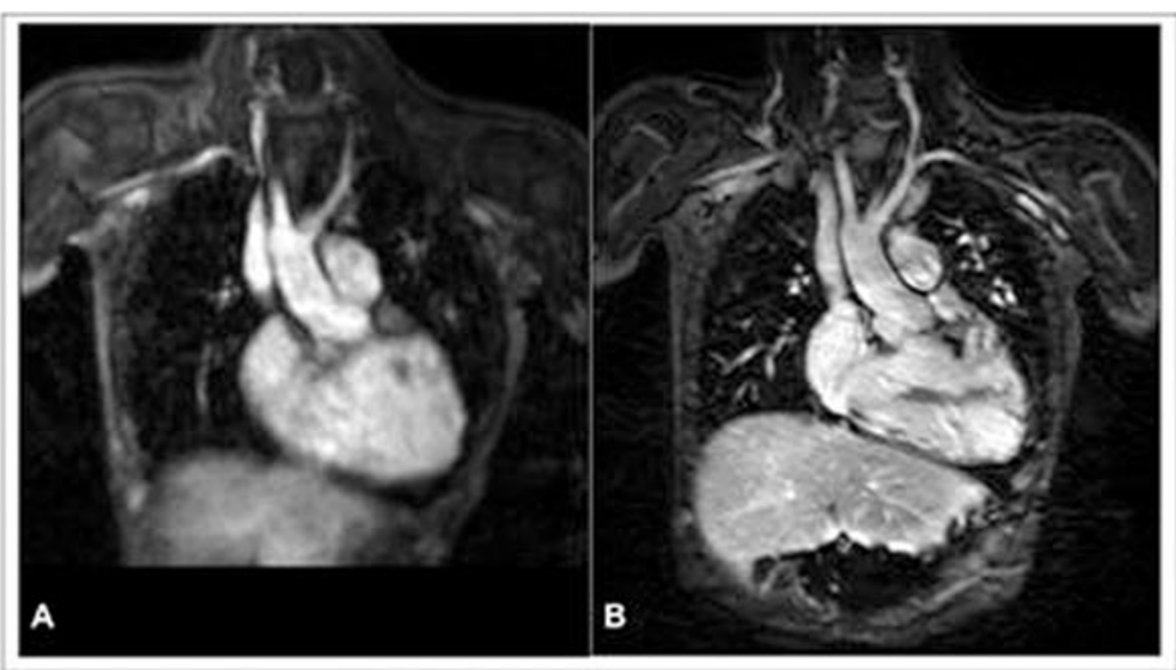

Figure 1 A: FF-MRA B: HR-MRA. 
FP-MRA and HR-MRA using an extracellular CA. HRMRA imaging was part of a comprehensive imaging protocol including viability imaging for assessment of $\mathrm{CD}$ and AD. Vessel sharpness of left pulmonary artery (LPA), left superior pulmonary vein (LSPV) and aorta ascendens (AA) were calculated. Furthermore, image quality was rated independently by two readers using a four point grading scale (1: no image artefacts and excellent vessel sharpness; 4) severe artefacts with poor vessel delineation).

\section{Results}

HR-MRA yielded significantly higher vessel sharpness for LSPV (FP-MRA: $0.38 \pm 0.11$ vs. HR-MRA: $0.57 \pm$ 0.12 ), LPA (FP-MRA: $0.37 \pm 0.06$ vs. HR-MRA: $0.50 \pm$ 0.11 ) and AA (FP-MRA: $0.50 \pm 0.10$ vs. HR-MRA: 0.74 $\pm 0.15)$ compared to the FP-MRA. Image quality was rated higher for HR-MRA compared to FP-MRA by both reviewers. Typical images are displayed in Figure 1, demonstrating the advantage of motion-compensated HR-MRA (B) vs. FP-MRA (A).

\section{Conclusions}

HR-MRA of the thoracic vasculature using extracellular CA offers superior vessel sharpness and improved image quality compared to standard FP-MRA at $3 \mathrm{~T}$. Thus HR-MRA may be used as an add-on to standard FPMRA. Due to the inherent delayed data acquisition of viability imaging in combination with double dose CA regimen, HR-MRA does not require additional scanning time or contrast agent injection.

Published: 2 February 2011

doi:10.1186/1532-429X-13-S1-P199

Cite this article as: Dabir et al:: First-pass and high-resolution ECG-gated MRA of the thoracic vasculature in children and adolescents using gadobutrol at 3 T. Journal of Cardiovascular Magnetic Resonance 201113 (Suppl 1):P199.
Submit your next manuscript to BioMed Central and take full advantage of:

- Convenient online submission

- Thorough peer review

- No space constraints or color figure charges

- Immediate publication on acceptance

- Inclusion in PubMed, CAS, Scopus and Google Scholar

- Research which is freely available for redistribution

Submit your manuscript at www.biomedcentral.com/submit 